\title{
Value of UVJ-M in the diagnosis of SUI in late pregnancy and postpartum
}

\author{
GUIXIN ZHANG $^{1}$, WEI JIANG ${ }^{1}$, QUANWEI GUO ${ }^{2}$ and QUANRONG GUO ${ }^{3}$ \\ Departments of ${ }^{1}$ Obstetrics and Gynecology, ${ }^{2}$ Ultrasonography, Tangshan Maternal and Child Health Center; \\ ${ }^{3}$ Emergency Department, Kailuan General Hospital, Tangshan, Hebei 063000, P.R. China
}

Received November 29, 2015; Accepted February 29, 2016

DOI: $10.3892 / \mathrm{etm} .2016 .3118$

\begin{abstract}
Stress urinary incontinence (SUI) is a common pelvic floor dysfunctional disorder in which leakage of urine occurs when there is abdominal pressure. The aim of the present study was to determine the value of stress urinary incontinences (SUIs) in late pregnancy and postpartum via detection of the mobility of the ureterovesical junction (UVJ-M) by using transperineal ultrasound. The study involved the continuous and random selection of 120 cases of early pregnant women and single births. The patients were divided into the SUI and non-SUI groups dependent on whether there was leakage of urine when abdominal pressure in the form of coughing, laughing and sneezing, was increased. UVJ-M was measured, the receiver operating characteristic (ROC) curve was drawn up and the threshold value was predicted. The results showed that, the SUI prevalence was $7.5(9 / 120)$, 22.5 (27/120), 43.3 (52/120), and 5.8\% (7/100), respectively, in 34,36 , and 38 gestational weeks, and 6 weeks after delivery. The SUI prevalence gradually increased with the gestational weeks, and differences were statistically significant. UVJ-M values increased with the gestational weeks, at $3.43 \pm 1.52$, $6.77 \pm 0.98$ and $2.35 \pm 1.04 \mathrm{~mm}$, respectively. Statistically significant differences were identified. Results of the ROC analysis, based on measurement of UVJ-M between the late pregnancy and non-SUI groups, revealed that the optimal threshold was $8.66 \mathrm{~mm}$, corresponding to a sensitivity of $89.5 \%$ and specificity of $66.7 \%$. In conclusion, UVJ-M $\geq 6.59 \mathrm{~mm}$ was identified as the predicted value of SUI during late pregnancy, and UVJ-M $\geq 8.66 \mathrm{~mm}$ the predicted value of SUI after delivery.
\end{abstract}

\section{Introduction}

Stress urinary incontinence (SUI) is a common pelvic floor dysfunctional disorder in middle-age women, accounting for

Correspondence to: Dr Quanwei Guo, Department of Ultrasonography, Tangshan Maternal and Child Health Center, 14 South Jianshe Road, Tangshan, Hebei 063000, P.R. China E-mail: goc364@163.com

Key words: transperineal ultrasound, mobility of urethrovesical junction, stress urinary incontinence, receiver operating characteristic curve
$>40 \%$ of cases in menopausal women (1). The disease involves leakage of urine when abdominal pressure due to coughing, laughing and sneezing occurs. Consequently, when the affected patients walk or lay down, urine leakage occurs. Since it is difficult to control urinary incontinence, many patients experience serious psychological barriers. Therefore, SUI, also known as the 'social cancer' constitutes both a health and social issue. The WHO has listed SUI as one of the five major chronic diseases (2). At present, the pathogensis of SUI remains to be determined although changes in the female pelvic floor and lower urethral continence-controlled system during pregnancy and childbirth constitute the primary risk factors for SUI (3).

Mobility of ureterovesical junction (UVJ-M), rotation angle and distance from the bladder neck to lower edge of pubic bone are largely associated with the occurrence and severity of SUI (4). Mouritsen and Rasmussen (5) suggested that an ultrasound diagnosis should be considered the reference standard. Pregazzi et al (6) suggested that UVJ-M >1 cm can be used as an objective indicator for the ultrasound diagnosis of SUI in non-pregnancy. In view of the non-invasive, no radiation, and repeatability of ultrasound diagnosis, the present study was conducted to predict the value of SUI in late pregnancy and postpartum by transperineal ultrasound. By using receiver operating characteristic (ROC) curve analysis, we obtained higher sensitivity and specificity of the threshold as an early screening of high-risk groups with SUI in late pregnancy and postpartum. Thus, to reduce the incidence of SUI and improve the quality of life of women in late pregnancy, early diagnosis, intervention and treatment are imperative.

\section{Patients and methods}

Patients. During the period December 2012-March 2014, 120 primigravidas with single birth were selected for the present study at the Tangshan Maternal and Child Health Center (Tangshan, China). Patients who experienced a mid-term induction of labor or maternal surgery, pre-existing SUI, body mass index $>30 \mathrm{~kg} / \mathrm{m}^{2}$, combined pregnancy hypertension, gestational diabetes mellitus, ultrasound examination of fetal weight $>4,000 \mathrm{~g}$ in the third trimester, too much or too little amniotic fluid, long-term constipation, chronic cough and asthma history were excluded. SUI diagnostic standards included leakage of urine when abdominal pressure was increased via coughing, laughing and sneezing, and use of the 

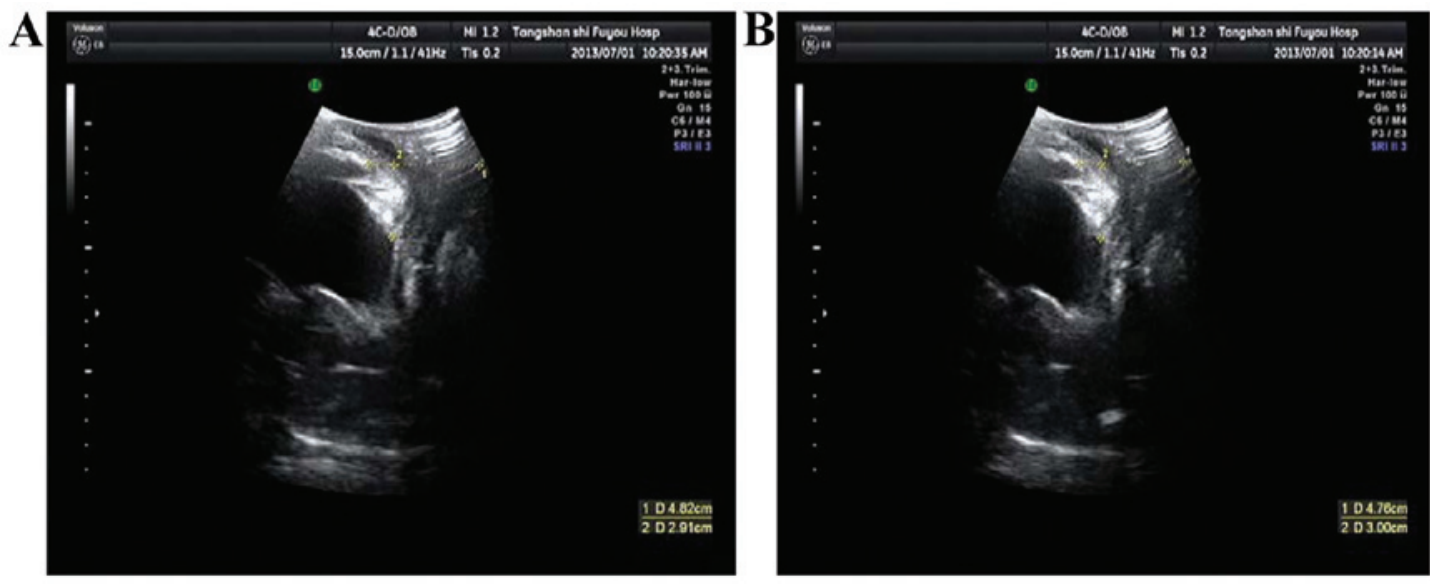

Figure 1. Non-stress urinary incontinence. (A) Quiescent condition, (B) valsalva condition.
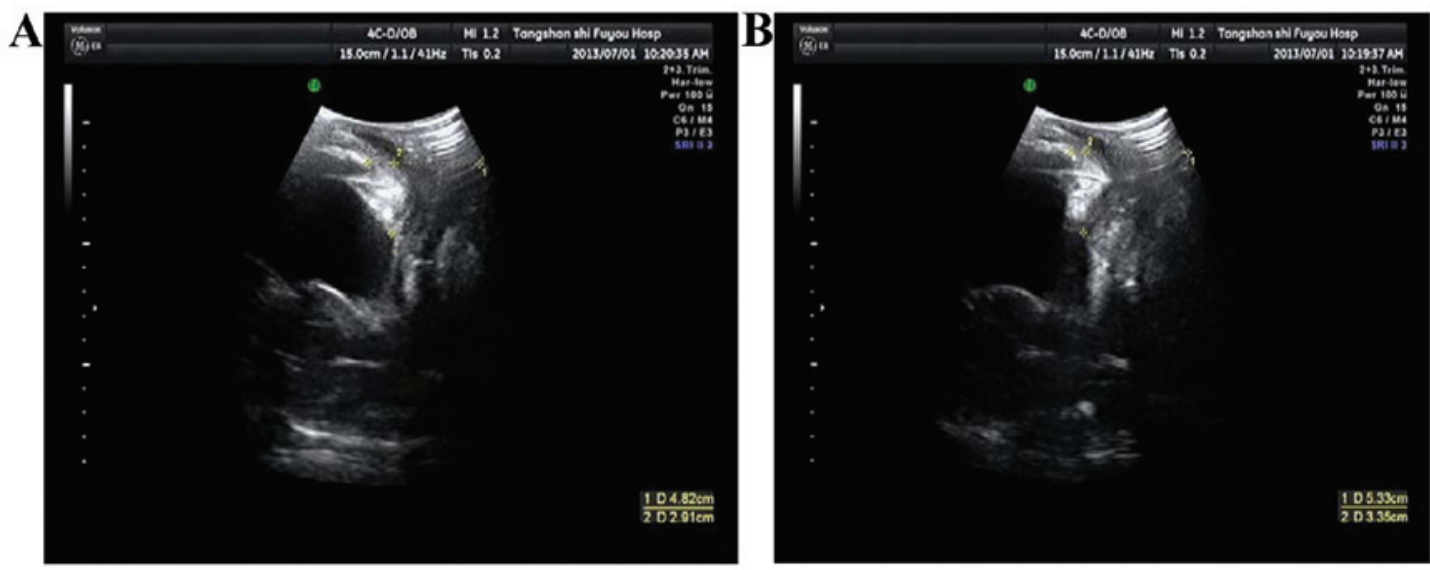

Figure 2. Stress urinary incontinence at 34 gestational weeks. (A) Quiescent condition, (B) valsalva condition.

international continence inquiring committee's questionnaire short form for accurate diagnosis.

Methods. Informed consent was obtained from patients and their families. The study was approved by the ethics committee of the Tangshan Maternal and Child Health Center (Hebei, China). Patients were divided into the SUI and non-SUI groups at 34,36 , and 38 gestational weeks and 6 weeks after delivery, respectively. The same color ultrasound room was fixed with GE Voluson E8 ultrasound equipment and the GE broadband convex array probe with frequency $5.0 \mathrm{MHz}$ (Willowick, $\mathrm{OH}$, USA), $15^{\circ}$ forward to the front-end probe with effective visual field of $90^{\circ}, 250 \mathrm{~mm}$ in length and diameter $17 \mathrm{~mm}$. The front end of the probe was slightly expanded with a maximum diameter of $27 \mathrm{~mm}$ (GE Healthcare, Piscataway, NJ, USA). An experienced sonographer measured the UVJ-M value, created the ROC curve and predicted the threshold value.

The UVJ-M measurement procedure required the patients be placed in a supine position. The bladder urine volume was evaluated as $250-500 \mathrm{ml}$ by regular color ultrasound. Patients were enjoined to bend knees in the lithotomy position. In addition, neutrally medical disinfection coupling agent should be smeared evenly on the surface of probe. Subsequently, the probe was covered with a condom on which neutrally medical disinfection coupling agent was applied. To place the probe into the perineum successfully, the axes of the handle of the probe were parallel to the vertical axes of the human body to ensure free movement of the probe. The investigator was required to use sufficient strength to overcome resistance of the vaginal walls and associated structures. The probe made longitudinal incisions and the investigator freeze-framed the images (sonogram), including lower margin of the pubic bone, base of bladder, UVJ, urethra, vagina and sagittal image of rectum. A trackball was used to draw up the outline of base of bladder and UVJ (7). During the entire procedure, the patient remained in a relaxed state. To obtain further images, Valsalva manoeuvres were carried out, the UVJ was observed to slide backward, a procedure also conducted for soft issues of variants and rectum. When UVJ-M reached its maximum, sonogram images were obtained again for measurement. By replaying the ultrasound image and moving the cursor, UVJ-M was measured as the distance between UVJ in stationary stage and UVJ in tension. The average of this distance was subsequently calculated. UVJ-M images of patients with or without SUI at different time points are shown in Figs. 1-5.

Observing index. To obtain the threshold of UVJ-M in late pregnancy and after delivery, the ROC curve was analyzed on the basis of SUI prevalence and UVJ-M value, respectively, in 34, 36 , and 38 gestational weeks and 6 weeks after delivery. ROC 

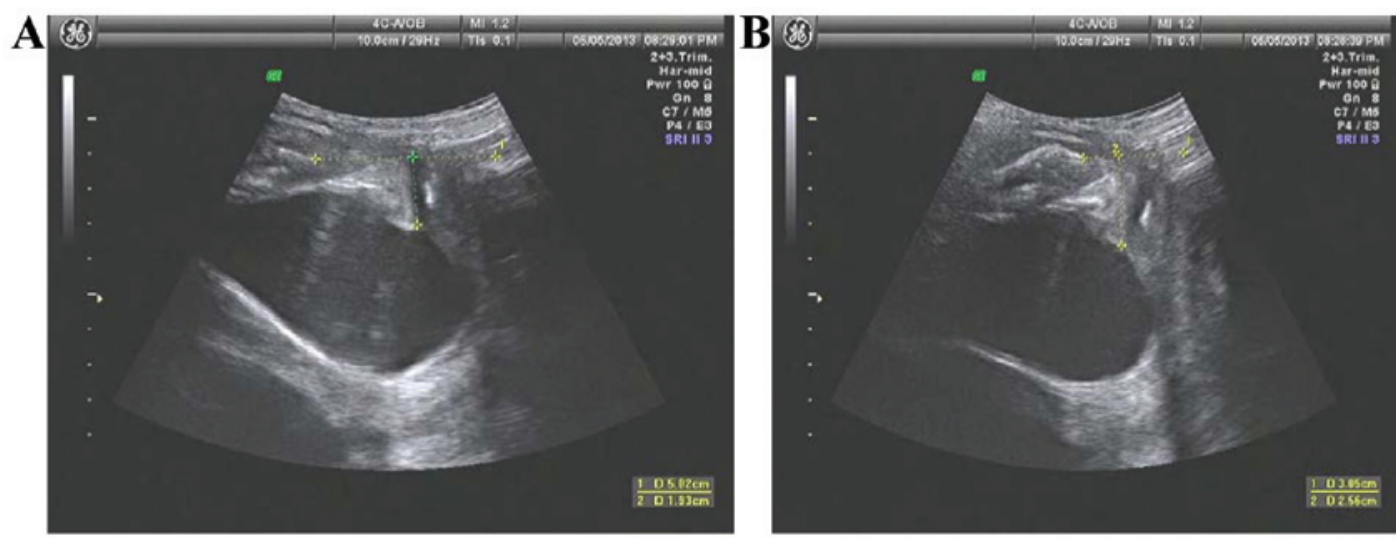

Figure 3. Stress urinary incontinence at 36 gestational weeks. (A) Quiescent condition, (B) valsalva condition.
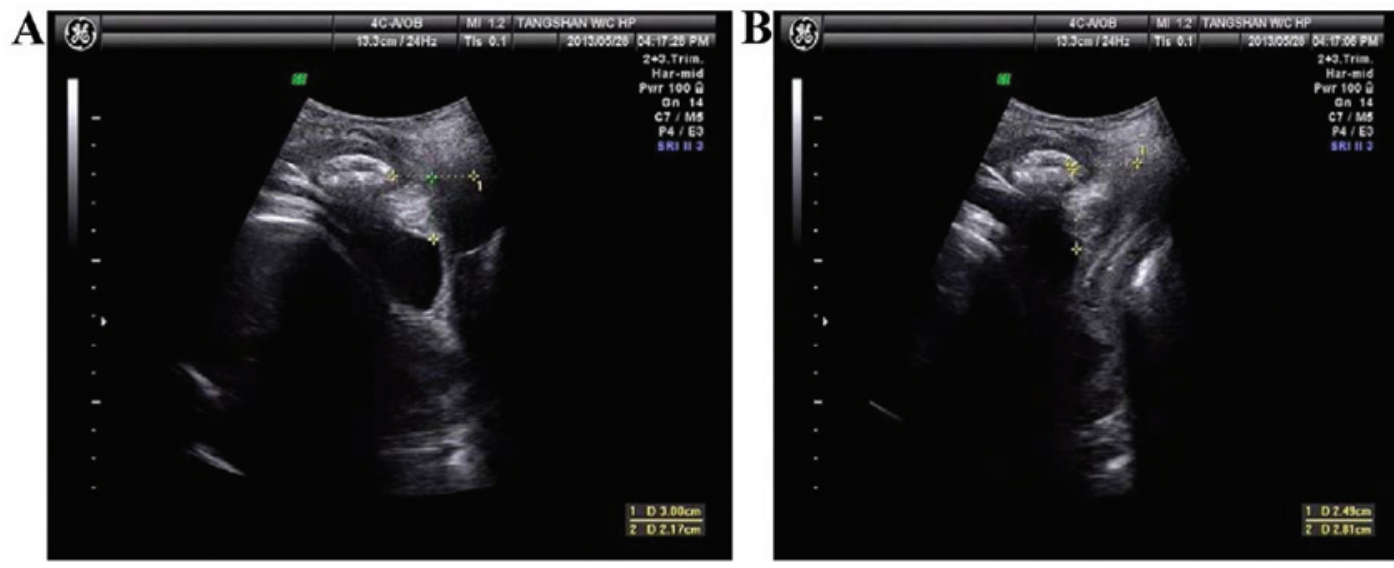

Figure 4. Stress urinary incontinence at 38 gestational weeks. (A) Quiescent condition, (B) valsalva condition.
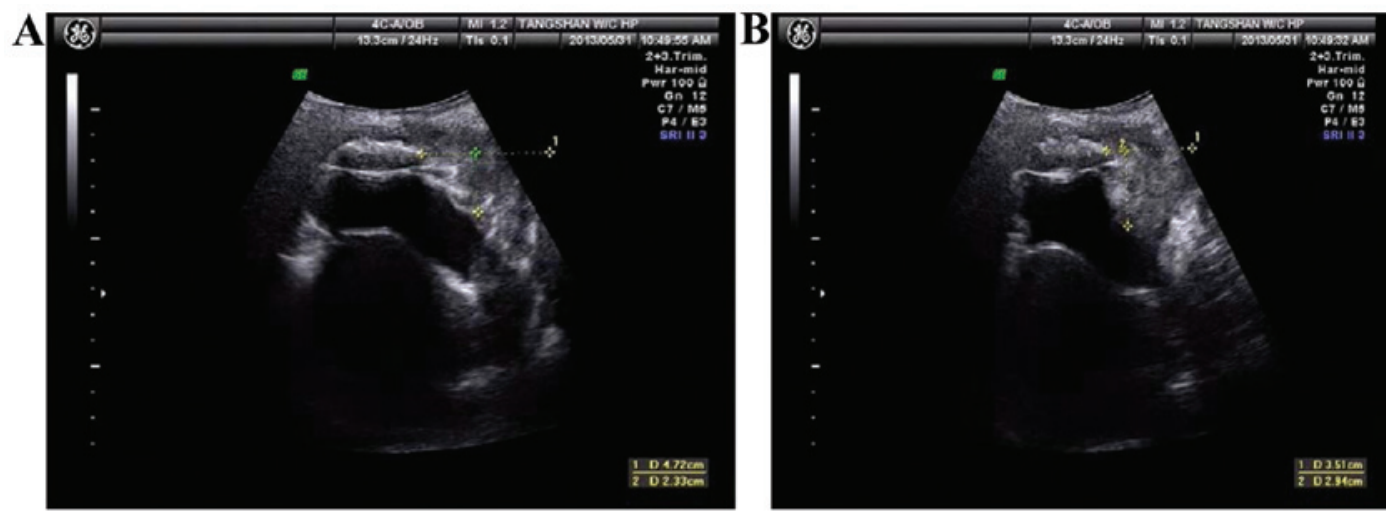

Figure 5. Stress urinary incontinence at 42 days after delivery. (A) Quiescent condition, (B) valsalva condition.

curve is dependent on true- and false-positive rates. Any point on the curve shows the relationship between the specific positive standard and the sensitivity and specificity in certain screening tests: UVJ-M value dring late pregnancy and postpartum (8).

Statistical analysis. Data were analyzed using SPSS 20.0 (IBM SPSS, Armonk, NY, USA) statistical software package. Measurement data were presented as mean \pm standard deviation. Comparisons in multiple and two groups were assessed using analysis of variance and independent sample t-test, respectively.
Measurement data were shown as [no. (\%)], and comparison between the groups was determined by the $\chi^{2}$ test. $\mathrm{P}<0.05$ was considered to indicate a statistically significant difference.

\section{Results}

Comparison of baseline data. Analysis of baseline data revealed that for 38 gestational weeks, there were 52 cases of SUI $(43.3 \%)$. No statistically significant differences were identified ( $\mathrm{P}>0.05)$ (Table I). 
Table I. Comparison of baseline data (mean \pm standard deviation).

\begin{tabular}{lccccc}
\hline Group & Case & Age & Height & BMI $\left(\mathrm{kg} / \mathrm{m}^{2}\right)$, before 34 gestational weeks & Growth in BMI $\left(\mathrm{kg} / \mathrm{m}^{2}\right), \mathrm{during}$ weeks \\
\hline SUI & 52 & $26.5 \pm 2.4$ & $159 \pm 18.2$ & $23.7 \pm 3.1$ & $6.5 \pm 1.1$ \\
Non-SUI & 68 & $25.8 \pm 2.6$ & $158 \pm 18.7$ & $23.5 \pm 3.2$ & $6.8 \pm 1.2$ \\
t-test & & 0.981 & 1.232 & 1.560 & 0.471 \\
P-value & & 0.423 & 0.924 & 0.785 & 0.326 \\
\hline
\end{tabular}

BMI, body mass index; SUI, stress urinary incontinence.

Table II. Different UVJ-M values in different stages of pregnancy.

\begin{tabular}{lcccc}
\hline Group & 34 weeks & 36 weeks & 38 weeks & $\begin{array}{c}6 \text { weeks } \\
\text { after delivery }\end{array}$ \\
\hline SUI & $3.43 \pm 1.52$ & $4.76 \pm 1.33$ & $6.77 \pm 0.98$ & $2.35 \pm 1.04$ \\
Non-SUI & $2.56 \pm 1.13$ & $3.76 \pm 1.48$ & $6.01 \pm 1.85$ & $1.41 \pm 0.66$ \\
t-test & 4.182 & 3.754 & 4.621 & 5.743 \\
P-value & 0.037 & 0.039 & 0.028 & 0.012 \\
\hline
\end{tabular}

UVJ-M, mobility of ureterovesical junction; SUI, stress urinary incontinence.

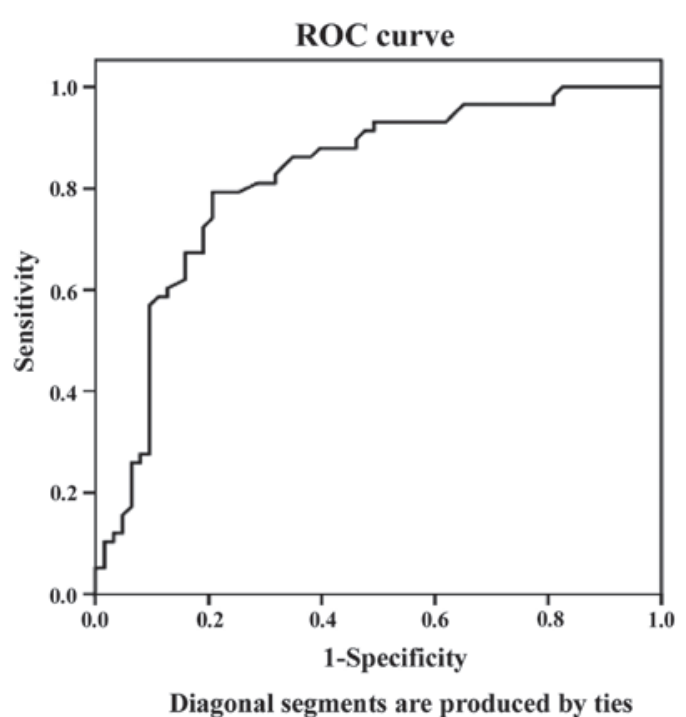

Figure 6. Analysis of receiver operating characteristic (ROC) curve of stress urinary incontinence and mobility of ureterovesical junction in late pregnancy.

SUI prevalence and UVJ-M in different stages of pregnancy. At 34, 36, and 38 gestational weeks, as well as 6 weeks after delivery, the SUI prevalence was 7.5 (9/120), 22.5 (27/12), $43.3(52 / 120)$, and $5.8 \%$ (7/120), respectively. The SUI prevalence was increased with the gestational weeks, with statistically significant differences $\left(\chi^{2}=5.624, \mathrm{P}=0.016\right)$ (data not shown). The UVJ-M value was $3.43 \pm 1.52,4.76 \pm 1.33$, $6.77 \pm 0.98$ and $2.35 \pm 1.04 \mathrm{~mm}$, respectively. The UVJ-M value increased with the gestational weeks, with a statistically significant difference (Table II).

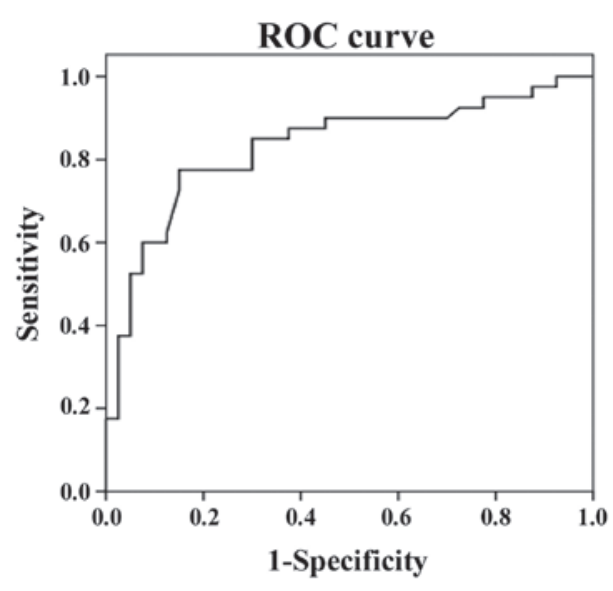

Diagonal segments are produced by ties

Figure 7. Analysis of receiver operating characteristic (ROC) curve of postpartum stress urinary incontinence and mobility of ureterovesical junction.

Analysis of ROC curve. ROC curve was constructed on the basis of UVJ-M whether SUI occurs in late pregnancy. Horizontal axes showed the specificity, and vertical axes showed the sensitivity. Generally, the point closest to the top left corner of the curve was considered the optimal threshold. Fig. 6 shows that the square below the curve was 0.819 . The standard error of the square was 0.039 , and the $95 \%$ confidence interval of the square was (0.742-0.896), with the exception of 0.5 . The ROC curve determined that the optimal threshold was $6.59 \mathrm{~mm}$, in correlation to a sensitivity of $95 \%$ and a specificity of $77.8 \%$.

The ROC curve analysis of UVJ-M from the SUI and non-SUI groups after delivery, revealed the square below the curve was 0.832 . The standard error of the square was 0.047 , and the $95 \%$ confidence interval of the square was $(0.739-0.925)$ with the exception of 0.5 . The ROC curve determined the optimal threshold to be $8.66 \mathrm{~mm}$ in correlation to a sensitivity of $89.5 \%$ and a specificity of $66.7 \%$ (Fig. 7).

\section{Discussion}

The pathogenesis of SUI remains to be determined. The hammock and integral hypotheses have been posited, with supporters of the hammock theory advocating that maintaining closure function of urethra is dependent on the pressure along the anterior wall of the vagina and the supporting structure of the bladder neck and the effective conduction between bladder neck and urethra (4). The pelvic floor compression of the urethra reduced the intensity and caused the occurrence of 
SUI, where the supporting structure of anterior vaginal wall and the bladder neck were destroyed. The integral theory of pelvic floor involves focuses on urinary bladder, urethra, uterus, rectum, anus, other pelvic organs and the related muscles as a whole (7). Any defects in part of the pelvic floor may lead to an imbalance of these interactions, resulting in urethral pressure not being effectively maintained when abdominal pressure is increased, leading to SUI. The occurrence of SUI is in affected by the anatomical position of the pelvic floor in both the hammock and integral hypotheses.

Pelvic floor damage in women is initiated during the gestational period. The incidence rate of SUI is not high during the early period of pregnancy. Owing to fetal growth and an increase in pressure, collagen fiber in the pelvic floor muscles is compelled to expand and stretch gradually, and tension relaxation occurs to meet the needs required for childbirth (8). However, 6 weeks after delivery, the muscles recover to the pre-pregnancy state, with the exception of breast. The location of pelvic organs is also recovered (9). Therefore, the ultrasound examination cannot only observe the location and mobility of the bladder neck change in late pregnancy with gestational weeks, but can evaluate recovery situations of position and function of pelvic organs. At present, a number of methods are available to examine SUI such as endoscopy, urethral pressure measurement, urine dynamics and magnetic resonance imaging. Of these tecniques, ultrasound, transperineal ultrasound in particular, allow for a real-time dynamic observation of the shape and position of the urethra, and bladder neck. Additionally, this ultrasound clearly identifies mutual associations between the various structures of the pelvic floor, thereby providing a morphological and functional basis for the clinical diagnosis and treatment of SUI. At the same time, advantages including non-radiation, safety, non-invasive, economic, single operation and good repeatability render it more suitable for pregnant women (10).

Parameters for ultrasonic monitoring of urinary incontinence includes reflecting the retrovesical angle of the posterior basal part in bladder and proximal urethra (11). It also includes vertical and horizontal distance in accordance with distance between bladder neck and symphysis. UVJ-M is an early parameter in the ultrasound examination of labia, which is considered an important parameter and significant sign of stability in bladder neck position. It is a major factor involved in female continence mechanism $(12,13)$. Furthermore, due to the overactivity of UVJ-M, the bladder neck junction is extremely low in tension, resulting in obstacles of abdominal pressure, leading to the occurrence of SUI in women (14-16). To construct the ROC curve and identify the threshold value predicted, the present study measured UVJ-M in 34,36 , and 38 gestational weeks and 6 weeks after delivery, respectively. SUI prevalence and UVJ-M were increased with the various gestational weeks. These differences were statistically significant. The predicted value of SUI in late pregnancy was UVJ-M $\geq 6.59 \mathrm{~mm}$, while that after delivery was UVJ-M $\geq 8.66 \mathrm{~mm}$.

The present study was based on the subjective symptoms of the patients. It mainly focused on late pregnancy and early postpartum. As the sample size in the present study was relatively small, additional samples and an extension of the follow-up time period to further confirm the reliability and accuracy of SUI in the diagnosis of particular intervals corresponding to UVJ-M during pregnancy and childbirth.

\section{Acknowledgements}

The study was supported by the Science and Technology Research and Development Support Program (Tangshan City, 2012). The correlation factors of urinary tract anatomy defects and postpartum SUI were indicated by no. 131302116z.

\section{References}

1. Abrams P, Andersson KE, Birder L, Brubaker L, Cardozo L, Chapple C, Cottenden A, Davila W, de Ridder D, Dmochowski R, et al; Members of Committees; Fourth International Consultation on Incontinence: Fourth International Consultation on Incontinence Recommendations of the International Scientific Committee: Evaluation and treatment of urinary incontinence, pelvic organ prolapse, and fecal incontinence. Neurourol Urodyn 29: 213-240, 2010.

2. Ge J, Lu YX, Zhang Z and Li X: The prevalence and cognition of urinary incontinence among female adults in Beijing. Chin J Chin J Clin Obstet Gynecol 11: 15-17, 2010.

3. Rinne KM, Kainulainen S, Aukee S, Heinonen S and Nilsson CG: Dynamic magnetic resonance imaging of the behavior of the mid-urethra in healthy and stress incontinent women. Acta Obstet Gynecol Scand 89: 373-379, 2010.

4. Kruger JA, Dietz HP and Murphy BA: Pelvic floor function in elite nulliparous athletes. Ultrasound Obstet Gynecol 30: 81-85, 2007.

5. Mouritsen L and Rasmussen A: Bladder neck mobility evaluated by vaginal ultrasonography. Br J Urol 71: 166-171, 1993.

6. Pregazzi R, Sartore A, Bortoli P, Grimaldi E, Troiano L and Guaschino S: Perineal ultrasound evaluation of urethral angle and bladder neck mobility in women with stress urinary incontinence. BJOG 109: 821-827, 2002

7. Cosimato C, Cipullo LM, Troisi J, Di Spiezio SA, Tommaselli GA, Oro RR, Zullo F, Altieri V and Guida M: Ultrasonographic evaluation of urethrovesical junction mobility: Correlation with type of delivery and stress urinary incontinence. Int Urogynecol J 26: 1495-1502, 2015.

8. Masata J, Svabik K, Martan A, Drahoradova P and Pavlikova M: [What ultrasound parameter is optimal in the examination of position and mobility of urethrovesical junction?]. Ceska Gynekol 70: 280-285, 2005.

9. Weinstein MM, Jung SA, Pretorius DH, Nager CW, den Boer DJ and Mittal RK: The reliability of puborectalis muscle measurements with 3-dimensional ultrasound imaging. Am J Obstet Gynecol 197: 68.e1-68.e6, 2007.

10. Minardi D, Piloni V, Amadi A, El Asmar Z, Milanese G and Muzzonigro G: Correlation between urodynamics and perineal ultrasound in female patients with urinary incontinence. Neurourol Urodyn 26: 176-182, discussion 183-184, 2007.

11. García SL, Ramírez DL, Rey JR, Calvo JF, Iglesias BR and Calvo AO: Complications of polypropylene mesh for the treatment of female pelvic floor disorders. Arch Esp Urol 64: 620-628, 2011.

12. Tincello DG, Botha T, Grier D, Jones P, Subramanian D, Urquhart C, Kirkemo A and Khandwala S; TVT Worldwide Registry Investigators: The TVT Worldwide Observational Registry for Long-Term Data: safety and efficacy of suburethral sling insertion approaches for stress urinary incontinence in women. J Urol 186: 2310-2315, 2011.

13. Chen Z, Chen Y, Du GH, Yuan XY, Wu J, Zeng XY, Hu ZQ, Cai D, Yang WM and Ye: Comparison of three kinds of mid-urethral slings for surgical treatment of female stress urinary incontinence. Urologia 77: 37-41, discussion 42, 2010.

14. DuBeau CE, Kuchel GA, Johnson T II, Palmer MH and Wagg A; Fourth International Consultation on Incontinence: Incontinence in the frail elderly: report from the 4th International Consultation on Incontinence. Neurourol Urodyn 29: 165-178, 2010.

15. McClurg D, Ashe RG and Lowe-Strong AS: Neuromuscular electrical stimulation and the treatment of lower urinary tract dysfunction in multiple sclerosis - a double blind, placebo controlled, randomised clinical trial. Neurourol Urodyn 27: 231-237, 2008.

16. Kim SO, Na HS, Kwon D, Joo SY, Kim HS and Ahn Y: Bone-marrow-derived mesenchymal stem cell transplantation enhances closing pressure and leak point pressure in a female urinary incontinence rat model. Urol Int 86: 110-116, 2011. 